\title{
Declarative, Temporal, and Practical Programming with Capabilities
}

\author{
William R. Harris*, Somesh Jha*, Thomas Reps ${ }^{* \dagger}$, Jonathan Anderson ${ }^{\ddagger}$, and Robert N. M. Watson ${ }^{\ddagger}$ \\ * $\{$ wrharris, jha, reps $\} @$ cs.wisc.edu; University of Wisconsin-Madison, Madison, WI, USA \\ ${ }^{\dagger}$ GrammaTech Inc., Ithaca, NY, USA \\ $\ddagger\{$ jonathan.anderson, robert.watson \}@cl.cam.ac.uk; University of Cambridge, Cambridge, England, UK
}

\begin{abstract}
New operating systems, such as the Capsicum capability system, allow a programmer to write an application that satisfies strong security properties by invoking securityspecific system calls at a few key points in the program. However, rewriting an application to invoke such system calls correctly is an error-prone process: even the Capsicum developers have reported difficulties in rewriting programs to correctly invoke system calls.

This paper describes capweave, a tool that takes as input (i) an LLVM program, and (ii) a declarative policy of the possibly-changing capabilities that a program must hold during its execution, and rewrites the program to use Capsicum system calls to enforce the policy. Our experiments demonstrate that capweave can be applied to rewrite security-critical UNIX utilities to satisfy practical security policies. capweave itself works quickly, and the runtime overhead incurred in the programs that capweave produces is generally low for practical workloads.
\end{abstract}

\section{INTRODUCTION}

Developing practical but secure programs remains a difficult, important, and open problem. Network utilities such as tcpdump and wget process data read directly from a network connection, but execute vulnerable code [1], [2]. File utilities and language interpreters are often run by a trusted user to process untrusted data, but also execute vulnerable code [3]-[7]. Once an attacker compromises vulnerable code in any of the above programs, he can typically perform any action allowed for the user that invoked the program.

Traditional operating systems provide only weak primitives for applications to manage their privileges. As a result, if a programmer wants to verify that his program is secure, he typically must first verify that the program satisfies very strong properties, such as memory safety. However, recent work [8]-[11] has produced new operating systems that allow programmers to develop programs that execute untrusted code yet satisfy strong security requirements. Moreover, programmers can develop such programs with much less effort than fully verifying the program for a traditional operating system. Such systems extend the set of system calls provided by a traditional operating system with security-specific calls (which henceforth we will call "security primitives"). Throughout a program's execution, it interacts with the system by invoking security primitives to signal key events in its execution. The developers of such systems have manually modified applications to invoke secu- rity primitives so that the application satisfies strong security policies, even when the application contains untrusted code.

One example of an operating system with strong security primitives is the capability operating system Capsicum [10], now an experimental feature in FreeBSD 9 [12]. Capsicum allows a programmer to compartmentalize his program into separate modules that each have a subset of the full set of privileges, following the principle of least privilege. Capsicum tracks for each process (1) the set of capabilities available to the process, where a capability is a file descriptor and an access right for the descriptor, and (2) whether the process has the privilege to grant to itself further capabilities (i.e., open more files). Capsicum provides to each process a set of system calls that the process invokes to limit its capabilities. Trusted code in a program can first communicate with its environment unrestricted by Capsicum, and then invoke primitives to limit itself to have only the capabilities that it needs for the remainder of its execution. Untrusted code then executes with only the limited capabilities defined by the trusted code. Thus, even if the untrusted code is compromised, it will only be able to perform operations allowed by the limited capabilities.

The Capsicum primitives are sufficiently powerful that a programmer can rewrite a practical program to satisfy a strong security policy by inserting only a few calls to Capsicum primitives [10]. However, in practice it is difficult for programmers to reason about the subtle, temporal effects of the primitives. When the Capsicum developers first evaluated Capsicum, they rewrote programs, such as tcpdump, in a way that they tentatively thought was correct, only to discover later that the program was incorrect and required a different rewriting [10]. Often, as in the case of tcpdump, the difficulty results from the conflicting demands of (i) using low-level primitives, (ii) ensuring that the program satisfies a strong, high-level security requirement, and (iii) preserving the core functionality of the original program.

This paper addresses the problem of writing programs for capability systems, like Capsicum, by presenting a system, called capweave, that takes from a programmer (1) a program that does not invoke Capsicum primitives, and (2) a declarative, temporal policy, stated in terms of the capabilities that the program should hold over the course of its execution, motivated by the principle of least privilege. capweave automatically compartmentalizes the program 
and instruments it to invoke Capsicum primitives so that it satisfies the policy when executed on Capsicum. We call the problem of finding such an instrumentation the Capsicum policy-weaving problem.

Our capweave policy weaver addresses two key challenges that a programmer faces when manually rewriting a program for Capsicum. The programmer's first challenge is to define what "secure behavior" means for his program. While Capsicum provides a powerful set of primitive operations, it does not provide an explicit language for describing policies. Because the Capsicum developers did not have such a language when first developing Capsicum, it was impossible for them to formally define correctness for their rewritten programs.

The programmer's second challenge is to write his program to be both secure and functional. A programmer can typically secure a program on Capsicum by strongly limiting the capabilities of the program. However, the rewritten program may limit its capabilities too strongly at one point of an execution, and as a result, may not have the capabilities required to carry out core program functionality later in the execution. The incorrect rewriting of tcpdump [10] exemplifies this issue. To resolve conflicts between security and functionality, a programmer must carefully rewrite his program to maintain additional state about an execution, and consult the state to determine when to invoke Capsicum primitives, or execute a program function in a separate process with distinct capabilities.

An additional challenge in designing capweave was to structure it so that it uses a simple, declarative model of Capsicum. Capsicum system architects and application developers have developed and continue to develop libraries of functions that an application can invoke to more easily manage its capabilities [13]. For the remainder of this paper, we refer to both the system calls and library functions that a program invokes to manage its capabilities as security primitives. When a Capsicum architect implements a new primitive, he should be able to easily extend capweave so that it can instrument programs to invoke the new primitive, but he should not need to understand the details of the instrumentation algorithm used by capweave.

To address the programmer's first challenge, capweave provides a policy language with which a programmer can write an explicit, declarative, general policy that restricts the privileges of the program in terms of capabilities. Each policy is a regular expression over an alphabet of program points paired with sets of capabilities. The policy allows all program executions that occur with the specified restricted privileges.

To address the programmer's second challenge, capweave takes an uninstrumented program and its policy, and automatically instruments the program to satisfy the policy. To do so, capweave constructs from the program, policy, and the semantics of Capsicum an automata-theoretic safety game [14] between an "Attacker," who "plays" program instructions, and a "Defender" who plays Capsicum primitives, by applying an automatatheoretic policy weaver [15]. The Attacker wins the game if the sequence of plays violates the policy, and the Defender wins otherwise. capweave searches for a winning Defender strategy, and from the strategy, instruments the program to (i) maintain instrumentation-state variables, and (ii) invoke Capsicum primitives based on the values of the variables so that the program satisfies its policy.

For a Capsicum architect to update capweave for an updated version of Capsicum, they only need to update declarative definitions of (i) the state maintained by Capsicum, (ii) the primitives available to a program, and (iii) each primitive's effect on the Capsicum state. In practice, the state and primitives are easy to define: together they account for only one tenth of the capweave source code.

We determined experimentally that capweave allows a programmer to harden practical programs to satisfy policies that rule out known critical exploits of the programs. We applied capweave to rewrite several UNIX utilities for Capsicum that have demonstrated security vulnerabilities. The rewritten programs included programs that were previously rewritten manually by the Capsicum team, programs suggested through discussion with the Capsicum development team, and the PHP CGI interpreter, whose policy was defined by independent security researchers at MIT Lincoln Laboratory. capweave allowed us to rewrite each utility using only a small handful of program annotations, no more than 11 lines, and a simple high-level policy of no more than 115 lines in our policy language. Each policy not only mitigated specific known exploits, but restricted the capabilities of significant segments of the program, potentially mitigating a large class of future vulnerabilities. Programs rewritten by capweave executed with equivalent behavior to programs instrumented manually by an expert, and incurred sufficiently low runtime overhead that they are still deployable: only $4 \%$ runtime overhead over unwoven programs on realistic workloads. We have provided a Capsicum virtual machine containing all programs and policies used in our experiments. ${ }^{1}$

Organization: $\S \mathrm{II}$ uses the wget downloader to illustrate the Capsicum policy-weaving problem and capweave. $\S$ III discusses the design of capweave in detail. $\S \mathrm{IV}$ presents an experimental evaluation of the correctness and performance of capweave and programs rewritten by applying capweave. $\S \mathrm{V}$ discusses related work.

\section{Motivation}

In this section, we motivate the Capsicum policy-weaving problem, and illustrate our solution by describing how capweave is used to secure the wget downloader.

\footnotetext{
${ }^{1}$ The virtual machine used is available at https://www.dropbox.com/s/ 711q31mccz47rt4/capweave-exp-vm.tar.gz.
} 


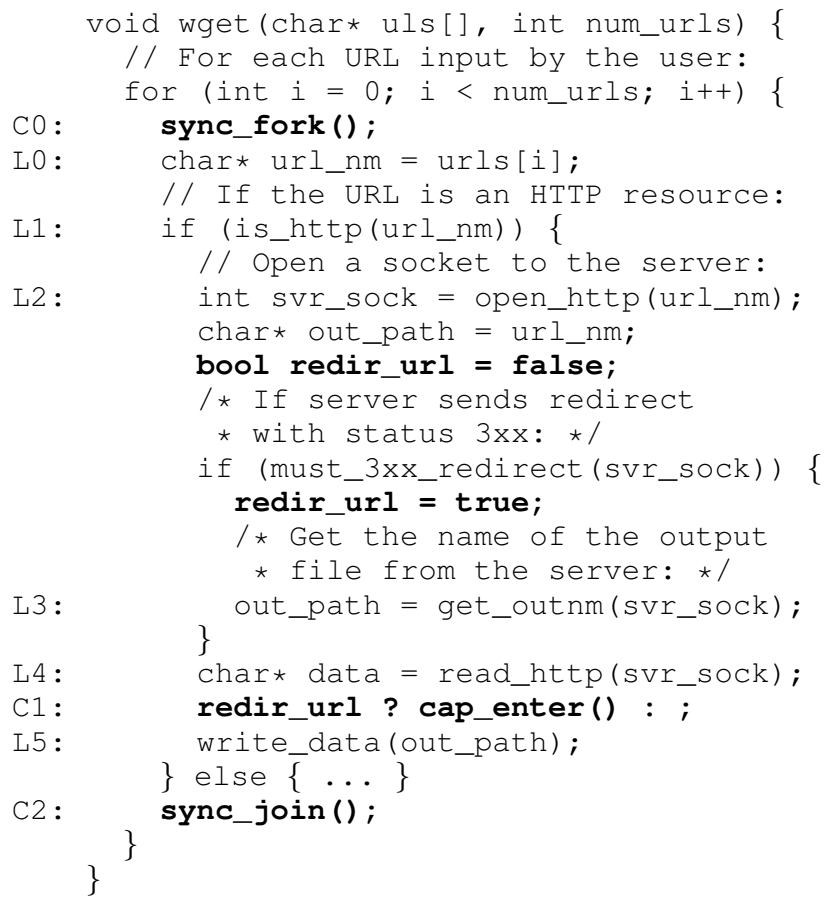

Figure 1. Pseudocode for the wget downloader, instrumented to invoke Capsicum primitives. wget takes an array of URL's as input, and writes the data at each URL to the file system of its host. Particularly subtle segments of wget's code are annotated with comments, and discussed in $\S$ II-A. Capsicum primitives are typeset in bold font.

\section{A. wget: an Insecure Program and a Desired Policy}

We now present a simplified version of the wget downloader and a desired security policy that past versions of wget do not satisfy. The wget downloader is a commandline utility that takes as input a list of URL's. For each URL, wget attempts to download the data addressed by the URL and write the data in the host file system.

Pseudocode for a simplified version of wget is given in Fig. 1. Important program points are annotated with $\mathrm{C}$ labels (e.g., L0). (Statements in Fig. 1 in bold font are invoked by a version of wget instrumented for Capsicum. Such statements are discussed in $\S$ II-B; for now, assume that wget does not execute such statements.) For each input URL, wget determines under what protocol the URL is addressed (Fig. 1, line L1). Once wget determines the protocol used, it runs protocol-specific functions to (i) open a socket to the server holding the URL (line L2), (ii) download the data addressed by the URL over the socket (lines L 3 and L4), and (iii) write the data to a file in the file system (line L5).

Unfortunately, versions of wget through v.1.12 include a vulnerability that allows an attacker who controls a server with which wget interacts to write data to any file on the host file system that can be written by the user who runs wget. The vulnerability is exposed when wget processes a particular HTTP response from the server. In particular, wget may receive from a server a redirect response, which directs wget to download data from a different network address. When wget receives such a response, it determines the path in its host file system to which it will write data directly from the information provided by the redirect server. A malicious server can exploit this behavior to craft a redirect response that causes wget to write data chosen by the attacker to a path in the file system chosen by the attacker [2].

Ideally, a wget developer would formally specify that wget must not demonstrate a vulnerability along the lines of the one described above, and would rewrite wget so that it satisfies such a specification. However, rewriting wget to do so requires detailed knowledge of both the structure of wget and of the HTTP protocol. Thus, it would be useful if a developer could define an acceptable, if perhaps weaker, specification for wget in terms of commonly-used, wellunderstood operating-system objects, such as file descriptors, and automatically rewrite wget to satisfy such a policy. In particular, one useful policy for wget defined in terms of file descriptors would be:

Policy 1. When wget executes read_http, it should always be able to open arbitrary files and sockets. But wget should execute write_data with the ability to open files if and only if it has not received an HTTP-redirect response.

\section{B. Securing wget on Capsicum}

The Capsicum operating system [10] provides a set of powerful security-oriented system calls (i.e., primitives) that an application can invoke to ensure that it only behaves in a secure manner, even if an attacker triggers a serious vulnerability in the application. Capsicum extends the notion of a file descriptor provided by UNIX to that of a capability by mapping each file descriptor opened by a process to a set of access rights that the process holds for the file descriptor. Each right corresponds roughly to the ability to perform a UNIX system call that operates on a file descriptor (e.g., the access right CAP_READ corresponds to the read system call). When a process running on Capsicum invokes a system call $\mathrm{C}$ on file descriptor $f$, Capsicum carries out $\mathrm{C}$ only if the process holds the right CAP_C for f. Capsicum also maps each executing process to an ambient-authority flag, which is a Boolean value that controls whether the process can open new file descriptors.

Capsicum's capabilities were designed so that a program executing on Capsicum begins by executing a small, trusted code segment that manages capabilities, and then executes complex, untrusted code that can interact with its environment only through the capabilities set by the trusted code. When a process opens a file descriptor, it holds all access rights for the descriptor. Throughout the process's execution, 
it can invoke a Capsicum primitive limitfd $(d, R)$ on descriptor $d$ and set of rights $R$ to decrease its rights for $\mathrm{d}$ to only those in $\mathrm{R}$. A process begins executing with the capabilities of its parent, and can invoke the Capsicum primitive cap_enter to relinquish the ambient authority.

A programmer can instrument wget to invoke the Capsicum primitives so that it satisfies Policy 1 (§II-A). One correct instrumentation of the example wget is the code shown in Fig. 1, including the Capsicum primitives shown in bold font. Essentially, wget is instrumented so that if it handles an HTTP redirection, then it invokes cap_enter before attempting to write data to its host's file system (line L5).

However, for a programmer to instrument his program to invoke Capsicum primitives correctly, he must address two challenges, illustrated by the instrumented version of wget. First, once a programmer formulates a policy, he must modify his program to invoke the Capsicum primitives to enforce the policy. However, the Capsicum primitives can have subtle consequences. In the example wget, once the programmer determines that under some conditions, wget should execute program point L5 without ambient authority, then the programmer can immediately deduce that wget must sometimes invoke the cap_enter primitive before executing L5. However, once the programmer also determines that if wget does not receive a redirect response, then wget should execute L5 with ambient authority, it is fairly difficult for him to instrument wget. The difficulty stems from the fact that once a process invokes cap_enter, then the process can never regain the ambient authority for the remainder of its execution. Thus, if a wget process (i) attempts to download from URL u, (ii) receives a redirection response, and (iii) invokes cap_enter, then the process must execute without ambient authority when downloading from all input URL's following u.

wget can be instrumented to satisfy the full informal policy of Policy 1 by compartmentalizing it to use multiple communicating processes. A "main" wget process executes the loop that iterates over the list of input URL's. To download data from each input URL, the main process synchronously forks a worker process to download the data and write it to the file system (line $\mathrm{CO}$ ). Each worker process begins executing with ambient authority. If the worker receives an HTTP-redirect response while downloading from its URL, then it invokes cap_enter, but when the worker process terminates (line $\mathrm{C} 1$ ), the main wget process continues to execute with ambient authority, with which it forks the next worker process. (Capsicum requires that a child process begin executing with the capabilities of its parent, but places no restrictions on the capabilities of the parent based on the capabilities of its children.)

Second, the instrumented program sometimes must update and consult additional instrumentation state to determine when to invoke Capsicum primitives. In Fig. 1, the instru-

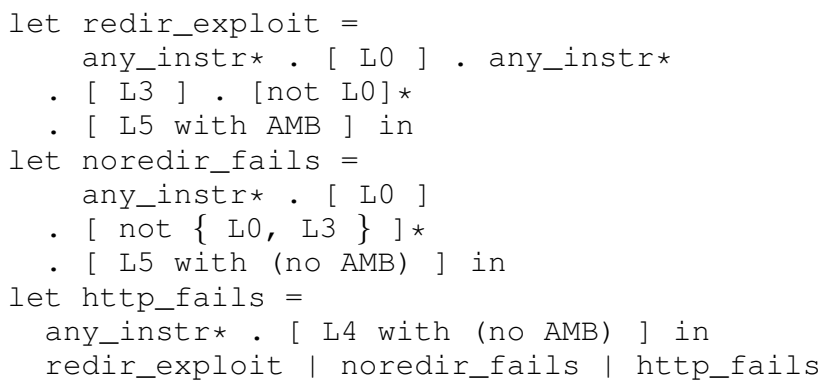

Figure 2. A capweave policy for the example wget given in Fig. 1. The policy is a regular expression that matches all undesired executions of wget, and is described in $\S \mathrm{II}-\mathrm{C}$.

mented wget maintains a Boolean variable redir_url that reflects whether or not wget received a redirection response when downloading from the current URL. The instrumented wget invokes cap_enter (line C1) if and only if redir_url is true.

Thus, a program can be rewritten to satisfy strong security requirements while preserving the functionality of the original program by correctly manipulating capabilities across multiple communicating processes and maintaining additional instrumentation state. However, it is non-trivial to determine how to rewrite an application to do so. In particular, the control locations at which an application must invoke primitives to satisfy a policy might not be near each other in the application's code. For example, in Fig. 1, wget invokes fork and cap_enter at distant program points.

\section{Securing wget on Capsicum with capweave}

$\S$ II-A and $\S$ II-B illustrate the general challenges that a programmer faces in rewriting a program to execute correctly on Capsicum. While a programmer can typically define the desired behavior of his rewritten program purely in terms of capabilities (e.g., Policy 1), Capsicum does not allow the programmer to state such a policy explicitly. Instead, the programmer must instrument his program manually to invoke primitives that manipulate both capabilities and processes so that the resulting program executes with the desired capabilities. To help a programmer address this challenge, we have developed a Capsicum policy weaver, called capweave. capweave takes as input from the programmer (1) a program that invokes no Capsicum primitives (for the example wget, the code in Fig. 1 without the instrumentation statements), and (2) a policy, which is a regular language of capability traces that each constitute a policy violation. A capability trace is a sequence of program points paired with the capabilities that the program has when it executes each program point.

A capweave policy that formalizes Policy 1 is given in Fig. 2. The language of violations in Fig. 2 is defined as the union of three sublanguages: redir_exploit, 
noredir_fails, and http_fails.redir_exploit formally expresses the set of all wget executions in which an attacker exploits wget's vulnerability in processing HTTP redirection responses. redir_exploit is defined as any sequence of instructions, followed by the program point at which the next URL in the array of inputs is selected ( $\mathrm{LO}$ ), followed by any sequence of instructions, followed by the program point at which wget processes an HTTP redirect response (L3), followed by any sequence of instructions before the selection of the next input URL (not LO), followed by wget writing downloaded data to the file system (L5) with ambient authority.

noredir_fails formally expresses the set of all wget executions in which wget does not receive an HTTP redirection response, but attempts to write downloaded data to the file system with insufficient rights. noredir_fails is defined as any sequence of instructions, followed by $\mathrm{L} 0$, followed by any sequence of instructions other than $\mathrm{L} 0$ or L3, followed by executing L5 with ambient authority.

http_fails formally expresses the set of all wget executions in which wget attempts to finish an HTTP session with insufficient rights. http_fails is defined as any sequence of instructions followed by attempting to complete the HTTP protocol (L4) without ambient authority.

For the simplified version of wget given in Fig. 1, the accompanying capweave policy given in Fig. 2 is almost as large as the program itself. However, in practice, policies for real-world programs tend to grow very slowly in the size of the program. The real wget program contains 64,443 lines of source code, but its entire policy can be expressed in only 35 lines of our policy language.

capweave outputs a version of the input program instrumented to invoke Capsicum primitives so that it satisfies the input policy. From the uninstrumented version of the example wget (i.e., Fig. 1 without the instrumentation statements) and the example policy given in Fig. 2, capweave outputs the correctly instrumented version of wget (Fig. 1 with the instrumentation statements).

\section{D. capweave Parametrized on the Capsicum Semantics}

The implemented version of capweave is actually structured slightly differently than described above: the implemented tool supports a more general model in which capweave is generated from an explicit description of the semantics of Capsicum [15]. Because of this model, capweave can be adapted easily when Capsicum is extended or when the "packaging" of sequences of invocations of Capsicum primitives as a library API is changed.

The Capsicum semantics defines (i) the state maintained by Capsicum as a program executes, (ii) the set of primitives that an instrumented program can invoke, and (iii) the effects of each primitive on the Capsicum state. In the current implementation of capweave, the state maintained by Capsicum is a stack of process states, where a process state is (a) a map from each descriptor to its current set of access rights, and (b) a Boolean value indicating whether the process has ambient authority. If a process state $p_{0}$ is below a process state $p_{1}$ on the stack, then the process whose state is $p_{0}$ spawned the process whose state is $p_{1}$ via a synchronous fork. The semantics also defines the effect of each primitive on the Capsicum state. For instance, cap_enter sets the Boolean value to False in the process state of the currently executing process (i.e., the top process on the stack); fork pushes a copy of the top process state onto the stack; join pops the top process state from the stack; etc.

It is significantly easier for a Capsicum architect to define a model of Capsicum using this mechanism than it would be for him to implement the entire policy weaver. capweave consists of 35k lines of OCaml that employs many subtle optimizations, whereas the Capsicum model is specified in only $3 \mathrm{k}$ lines, which essentially define a Capsicum interpreter. (The Capsicum state and interpretation functions are discussed in more detail in $§$ III-A3.)

In general, the Capsicum semantics would be specified by a Capsicum architect, rather than an application implementer, and would be changed rarely-either when new Capsicum primitives are introduced or when there are changes in the API of a library that packages Capsicum calls into routines that are more convenient to use than "raw" Capsicum. Application programmers can then regenerate an updated capweave tool and weave policies into as many applications as they wish.

\section{Design OF THE POLICY WeAVER}

\section{A. The Policy-Weaving Problem}

1) Language Syntax: The syntax of languages of both unwoven and woven programs will be defined as instances of a language of simple imperative programs, IMP. IMP is a small "core" language that supports only updates to program state with the result of language operations, operations on descriptors, invocations of woven instructions, and conditional branches of control-flow. However, the actual implementation of capweave instruments programs in the LLVM intermediate language [16], and thus can weave programs compiled from widely-used, practical languages, such as $\mathrm{C}$ and $\mathrm{C}++$.

Syntax of Unwoven Programs: The syntax of language IMP /wvinstrs (Fig. 3) is defined for a fixed set of program variables Vars, a fixed set of control labels Labels, and a set of open sites Opens that label program instructions at which descriptors are opened. The syntax is parametrized on a set of woven instructions wvinstrs (i.e., security primitives). An IMP program prog is a set of instruction blocks, including an

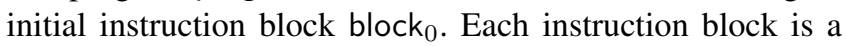
unique label, a statement, and a block-terminator instruction. A statement either assigns the result of a language operation to a variable, opens a descriptor, or executes a weaving instruction. A block terminator halts the program 


$$
\begin{aligned}
\operatorname{prog}:= & \left(\text { block }_{0},\left\{\text { block }_{1}, \ldots, \text { block }_{n}\right\}\right) \\
\text { block }:= & \text { LABEL }: \text { stmt } ; \text { termin } \\
\text { stmt }:= & \mathrm{v}_{0}:=\operatorname{op}\left(\mathrm{v}_{1}, \ldots, \mathrm{v}_{n}\right) \\
& \mid \text { dscinst } \\
& \mid \text { wvinstrs } \\
\text { termin }:= & \text { halt } \mid \text { br } \mathrm{v} ? \mathrm{v}_{i} \in \text { LABEL } \\
\text { dscinst }:= & \text { os }: \mathrm{v}_{0}:=\text { open }\left(\mathrm{v}_{1}\right), \\
& \mathrm{v}_{0}, \mathrm{v}_{1} \in \text { Vars, os } \in \text { Opens }
\end{aligned}
$$

Figure 3. Syntax of the IMP/wvinstrs〉 language: an imperative language parametrized on a set of woven instructions wvinstrs.

$$
\begin{array}{rlr}
\text { capinstr }:= & \mathrm{v}_{0}:=\operatorname{op}\left(\mathrm{v}_{1}, \ldots, \mathrm{v}_{n}\right) & \mathrm{v}_{i} \in \mathrm{W} \text { Vars } \\
& \mid \mathrm{v} ? \text { capprim } & \mathrm{v} \in \mathrm{W} \text { Vars } \\
\text { capprim }:= & \text { cap_enter } & \\
& \mid \text { limitfd(os, rs }) & \text { os } \in \text { Opens, rs } \subseteq \text { Rights } \\
& \mid \text { fork } & \\
& \mid \text { join } &
\end{array}
$$

Figure 4. Syntax of the set of Capsicum woven instructions capinstr.

or branches. The language of unwoven programs UNWOVEN is the language of imperative programs with no woven instructions: UNWOVEN $=\operatorname{IMP}\langle\emptyset\rangle$.

Syntax of Woven Programs: The language of woven programs is the language of IMP programs that may execute Capsicum woven instructions (Fig. 4), defined over a set of weaving variables WVars. A Capsicum woven instruction may assign the result of a language operation to a variable in WVars, or may execute a guarded invocation of a Capsicum primitive. A Capsicum primitive is either cap_enter, fork, join, or limitfd(os, rs), for os $\in$ Opens and rs $\subseteq$ Rights. A woven program is an IMP program instrumented to execute Capsicum instructions: WOVEN $=\mathrm{IMP}\langle$ capinstr $\rangle$.

2) Language Semantics: In this section, we define a semantics of WOVEN programs by mapping each WovEN program to the executions that it may perform. In particular, we define a semantic function $\tau$ that maps every Woven program $P$ and initial program state $p$ to the trace of capabilities that $P$ holds throughout its execution from $p$. $\tau$ is defined using an operational-semantic function $\sigma_{s}$ that describes how each program statement updates the state of the program. $\sigma_{s}$ is defined using the operational-semantic function $\sigma_{w}$, which define how each weaving instruction updates the program state. $\sigma_{w}$ is defined using the Capsicum interpretation of Capsicum primitives, which defines how each primitive updates the state maintained by Capsicum (§III-A3).

The semantics of Woven, given in Fig. 5, is defined by a function $\tau$ (Fig. 5, Eqns. (1) and (2)) that maps each program in WOVEN and initial program state $p$ to the sequence of capabilities that the program holds during an execution that starts from $p$. Let a program state be an assignment from each program variable to an integer value: progstates $=$ Vars $\rightarrow \mathbb{Z}$, where $\mathbb{Z}$ denotes the set of integers. A capability state is the state maintained by the Capsicum operating system. The set of capability states capstates is defined by the Capsicum architect (see §IIII-A3). Let a capability trace be a sequence of program labels paired with the capabilities that the program has as it executes the block with the given label: captraces $=$ (Labels $\times$ capstates $)^{*}$. $\tau$ maps each program $P \in$ Woven and program state $p \in$ progstates to the capability trace that $P$ generates in an execution that starts from $p$ (Fig. 5, Eqn. (1)). The trace generated by $P$ from a program state $p$ is the trace that the initial block of $P$ generates in an execution that starts from $p$, along with a fixed initial woven state and capability state (Fig. 5, Eqn. (2)).

The semantic function $\tau_{b}$, given in Fig. 5, Eqns. (3) and (4), defines the capability trace that a block generates in an execution from a given state. Let a woven state be a map from each weaving variable to an integer value: wvstates $=$ WVars $\rightarrow \mathbb{Z}$. Let a full state be a programstate, woven-state, and capability-state triple: fullstates = progstates $\times$ wvstates $\times$ capstates. Then $\tau_{b}$ defines the capability trace generated by executing a given block from a given full-state (Fig. 5, Eqn. (3)). The trace generated by executing a block LABEL : $s ; t$ from a full state $f$ is LABEL paired with the capability state in $f$, followed by the trace generated by executing the terminator instruction $t$ starting in the full-state obtained by updating $f$ with the statement $s$ (Fig. 5, Eqn. (4)).

The terminator semantic function $\tau_{t}$, given in Fig. 5, Eqns. (5)-(7), defines the trace generated by executing a block terminator from a given full-state $f \in$ fullstates. The terminator halt generates the empty trace (Fig. 5, Eqn. (6)). The terminator br $\mathrm{v}$ ? $\mathrm{LABEL}_{t}: \mathrm{LABEL}_{f}$ generates the trace obtained by executing either the block labeled $\mathrm{LABEL}_{t}$ or the block labeled $\mathrm{LABEL}_{f}$ from full-state $f$, depending on whether v's value is non-zero or zero, respectively (Fig. 5, Eqn. (7)).

The statement semantic function $\sigma_{s}$, given in Fig. 5, Eqns. (8)-(11), defines how a statement $s \in$ stmt updates a full-state $f \in$ fullstates. If $s$ is an assignment, then it updates the program state in $f$ according to the semantics of the right-hand-side operation (Fig. 5, Eqn. (9)). In Eqn. (9), the language semantics is denoted by the function $\iota_{\mathrm{o}}:$ stmt $\rightarrow$ progstates $\rightarrow$ progstates, and omitted for brevity. If $s$ is a descriptor instruction, then it updates the program and capability state in $f$ according to the Capsicum interpretation of descriptor instructions $\iota_{\mathrm{d}}$ (Fig. 5, Eqn. (10); for a discussion of $\iota_{\mathrm{d}}$, see $\S$ III-A3). If $s$ is a weaving instruction, then it updates the weaving and capability state in $f$ as defined by the weaving-instruction semantic function $\sigma_{w}$ (Fig. 5, Eqn. (11)). 


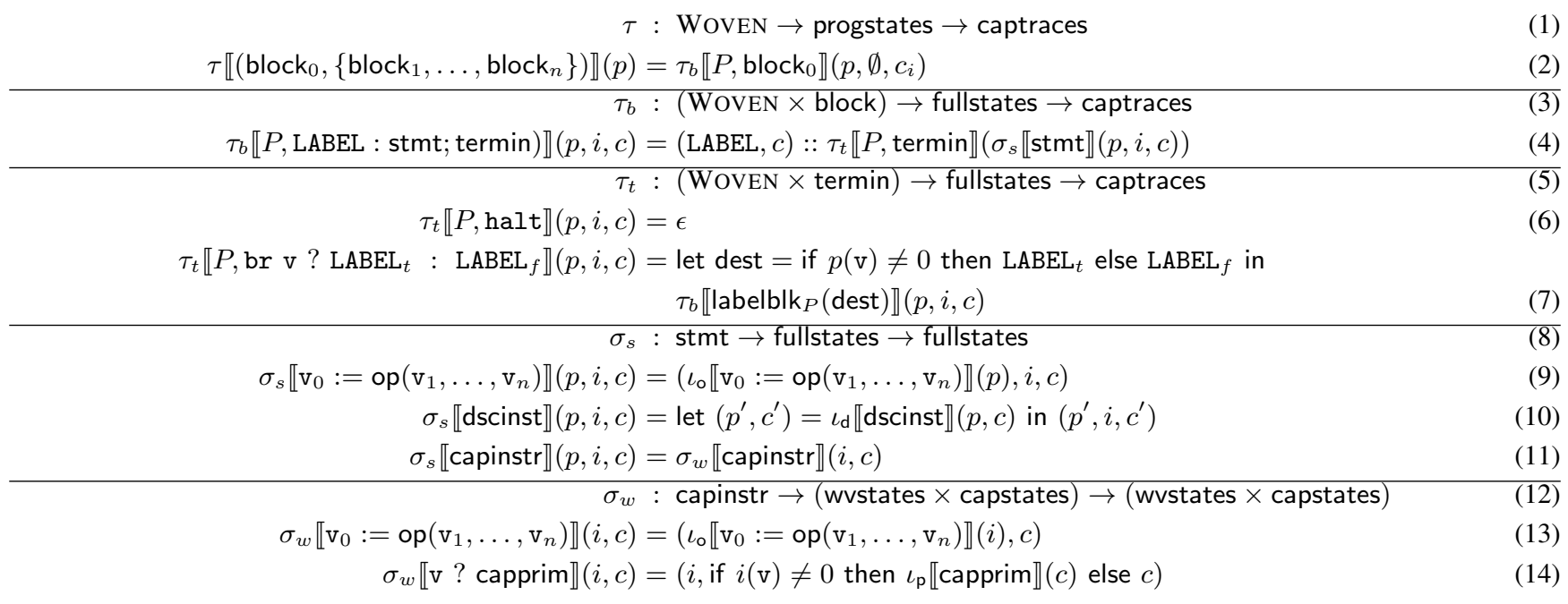

Figure 5. Semantics of Woven. $\tau, \tau_{b}$, and $\tau_{t}$ define the capability trace that a WovEN program generates by executing a given program, block, or terminator instruction, respectively, from a given state. In the definition of $\tau_{t}$, labelblk ${ }_{P}$ maps each label to the instruction block that it labels. $\sigma_{s}$ and $\sigma_{w}$ define how a program statement and woven instruction, respectively, update the state of a program. $\iota_{\mathrm{o}}$ and $\iota_{\mathrm{p}}$ denote the interpretation of program operations and Capsicum primitives (Fig. 6), respectively). progstates, wvstates, capstates, and fullstates denote the spaces of program states, woven states, capability states, and "full" program states, respectively (see §III-A2).

The woven-instruction semantic function $\sigma_{w}$, given in Fig. 5, Eqns. (12)-(14), defines how a woven instruction $v \in$ wvinstrs updates a woven state $w \in$ wvstates and a capability state $c \in$ capstates. If $v$ stores the value of a language operation in a woven-state variable, then the woven state is updated according to the language semantics (Fig. 5, Eqn. (13)). If the woven instruction is a guarded Capsicum primitive $\mathrm{v} ? \mathrm{p}$, then if $\mathrm{v}$ is zero in $w, \mathrm{v}$ ? $\mathrm{p}$ does not update the woven state, and otherwise, $\mathrm{v}$ ? $\mathrm{p}$ updates $c$ according to the Capsicum interpretation of primitive $p$ (Fig. 5, Eqn. (14); for a discussion of the interpretation of primitives $\iota_{\mathrm{p}}$, see §III-A3).

3) Capsicum Interpretation Functions: The semantics of Woven (§III-A2) is defined from (1) the space of capability states maintained by Capsicum, (2) the initial capability state with which a program executes, and (3) the Capsicum interpretations, which define how program instructions and Capsicum primitives update capability states. If the semantics of Capsicum were to be extended or revised in some way, these are the only pieces of information that a Capsicum architect would have to modify to obtain an updated version of capweave.

A capability state defines what capabilities are held by a program (Fig. 6, Eqns. (15) and (16)). Let a process capability state be a Boolean, denoting whether a process has ambient authority, together with a map from each descriptor to a set of rights (Fig. 6, Eqn. (15)). A capability state is a stack of process capability states (Fig. 6, Eqn. (16)).

The initial capability state $c_{i}$ is a singleton stack containing a process state denoting that the process has ambient capability, and that the rights of no descriptors are defined: $c_{i}=[($ True,$\emptyset)]$.

The Capsicum interpretation functions are given in Fig. 6. The first interpretation function $\iota_{\mathrm{d}}$ defines how each descriptor instruction $i \equiv \mathrm{d}: \quad \mathrm{x}:=$ open (y) (Fig. 6, Eqns. (17) and (18)) updates a program state $p \in$ progstates and capability state $c \in$ capstates (Fig. 6, Eqn. (17)). If the program holds ambient authority in $c$, then $i$ updates $p$ so that $\mathrm{x}$ holds a fresh descriptor, and updates $c$ so that the fresh descriptor has all access rights. Otherwise, $i$ updates $p$ so that $\mathrm{x}$ holds the value -1 , and the latest descriptor opened at $\mathrm{d}$ is not mapped to any set of access rights (Fig. 6, Eqn. (18)).

The second interpretation function $\iota_{\mathrm{p}}$ (Fig. 6, Eqns. (19) and (20)) specifies how a Capsicum primitive $p \in$ Capprims updates a capability state $c \in$ capstates (Fig. 6, Eqn. (19)). If a program executes cap_enter, then the program relinquishes ambient authority (Fig. 6, Eqn. (20)). If a program invokes $\operatorname{limitfd}(d, R)$, then the program's rights for the last descriptor opened at $d$ are updated to the intersection of the program's rights in $c$ and the set of rights $R$ (Fig. 6, Eqn. (21)). If a program invokes fork, then the program pushes a copy of the current process-capability state onto the stack of process capability states (Fig. 6, Eqn. (22)). If a program invokes join, then the program pops its top process capability state $p c$, and updates the new top process state in its capability state to have the descriptor rights in $p c$ (Fig. 6, Eqn. (23)).

Policy Semantics of Woven: A policy is a set of executions of a program annotated with the capabilities that the program must have as it executes. Although the capability 


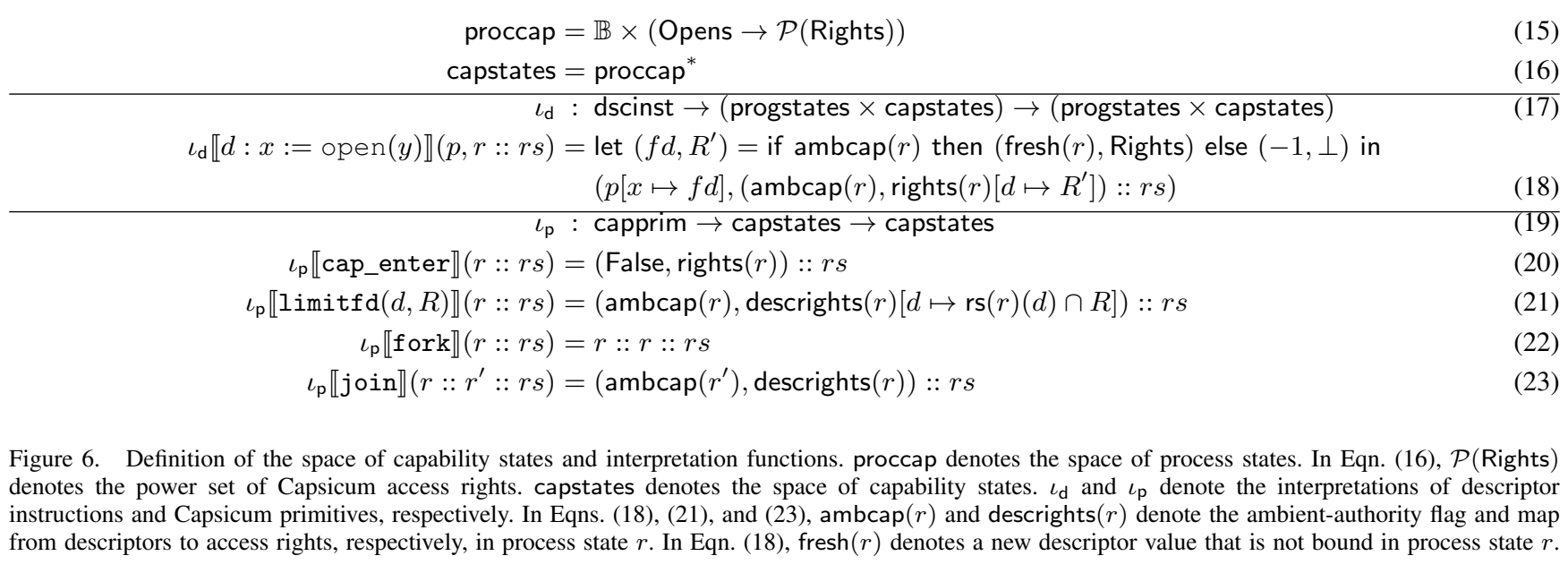

state of a program completely defines the capabilities held by a program as it executes, writing policies defined by the complete capability-state may be complicated or infeasible. In particular, the Capsicum interpretation functions in Fig. 6 are defined over capability states that are stacks of process capability states, but practical policies are typically defined over only the currently executing process (i.e., the top process on the stack of process capability states).

To bridge the gap between the capability state maintained by Capsicum and the state used to define policies, the Capsicum architect defines a space of policy states polstates and a policy-state abstraction $\alpha$ : capstates $\rightarrow$ polstates that maps each capability state to the policy state that represents it. Our implementation of capweave allows policies to be defined using the capabilities of the currently executing process: polstates $=$ proccap, and $\alpha$ (cur :: procs $)=$ cur.

A policy-state abstraction $\alpha$ defines a policy-semantics function that maps each woven program and initial program state to the trace of program labels paired with policy states that the program generates in an execution from the initial program state. For poltraces $=(\text { Labels } \times \text { polstates })^{*}$, the policy semantics function $\tau_{\alpha}$ : Woven $\rightarrow$ (progstates $\rightarrow$ poltraces) is

$$
\tau_{\alpha} \llbracket \operatorname{prog} \rrbracket(p)=m_{\alpha}(\tau \llbracket \operatorname{prog} \rrbracket(p))
$$

where

$$
\begin{aligned}
m_{\alpha}(\epsilon) & =\epsilon \\
m_{\alpha}((\text { LABEL }, c):: t) & =(\text { LABEL }, \alpha(c)):: m_{\alpha}(t)
\end{aligned}
$$

4) Problem Definition: The policy-weaving problem is to take an unwoven program and a policy, and weave the program to satisfy the policy. We formally define the weaving problem using the policy semantics of a program and the definition of a weaving. To simplify the definition of the policy-weaving problem, we fix the definition of the Capsicum interpretation functions, initial state, and policystate abstraction to be as defined in Fig. 6 and §III-A2. The definition of the program and policy semantics of Woven programs is thus fixed as well.

For an unwoven program $P \in$ UNWOVEN and a woven program $P^{\prime} \in$ Woven, $P^{\prime}$ is a weaving of $P$ if $P^{\prime}$ is constructed by only adding woven instructions to $P$.

Defn. 1. For IMP statements $s$ and $s^{\prime}, s^{\prime}$ is a weaving of $s$ if one of the following holds:

- $s$ is not a sequence of statements, and (1) $s^{\prime}=s$ or (2) $s^{\prime}=s_{0}^{\prime} ; s_{1}^{\prime}$ and either $s_{0}^{\prime}$ is a weaving of $s$ and $s_{1}^{\prime}$ is a sequence of woven instructions, or $s_{0}^{\prime}$ is a sequence of woven instructions and $s_{1}^{\prime}$ is a weaving of $s$.

- $s$ is a sequence of statements $s_{0} ; s_{1}$ and $s^{\prime}$ is a sequence of statements $s_{0}^{\prime} ; s_{1}^{\prime}$ where $s_{0}^{\prime}$ is a weaving of $s_{0}$ and $s_{1}^{\prime}$ is a weaving of $s_{1}$.

A program $P^{\prime}=\left(\right.$ block $_{0}^{\prime},\left\{\right.$ block $_{1}^{\prime}, \ldots$, block $\left.\left.{ }_{n}^{\prime}\right\}\right)$ is a weaving of a program $P=\left(\right.$ block $_{0},\left\{\right.$ block $_{1}, \ldots$, block $\left.\left._{n}\right\}\right)$ if for each $0 \leq i \leq n$, block $_{i}=\mathrm{LABEL}_{i}: s_{i} ; t_{i}$ and block $_{i}^{\prime}=\mathrm{LABEL}_{i}: s_{i}^{\prime} ; t_{i}$, where $s_{i}^{\prime}$ is a weaving of $s_{i}$.

The policy-weaving problem is to take an unwoven program and a policy defining the allowed executions of the program, and instrument the program so that it satisfies the policy.

Defn. 2. Let $P \in \mathrm{UNWOVEN}$ be an unwoven program, and let $Q \subseteq$ captraces be a regular language of capability traces. For a woven program $P^{\prime}$, let the traces of $P^{\prime}$, denoted as $\mathcal{T}\left(P^{\prime}\right) \subseteq$ captraces, be the set of capability traces generated by some input to the program: $\mathcal{T}\left(P^{\prime}\right)=\left\{\tau_{\alpha} \llbracket P^{\prime} \rrbracket(i) \mid i \in\right.$ $(\operatorname{Vars} \rightarrow \mathbb{Z})\}$. A solution to the policy-weaving problem $\operatorname{Weave}(P, Q)$ is a woven program $P^{\prime} \in$ Woven such that $P^{\prime}$ is a weaving of $P($ Defn. 1$)$ and $\mathcal{T}\left(P^{\prime}\right) \subseteq Q$.

WEAVE is undecidable in general; it can be shown that any algorithm that could solve WEAVE could decide if a program in a Turing-complete language satisfies an arbitrary safety property. capweave uses a sound but incomplete 
solver for WEAVE, described in $\S$ III-B.

\section{B. Solving the Policy Weaving Problem via Automata Games}

capweave reduces WEAVE to finding a winning strategy to a two-player safety game, played by an Attacker and a Defender. capweave uses an existing automata-theoretic weaver-generator algorithm [15] as its core engine. To make the paper self-contained, this section summarizes that algorithm, and describes how capweave applies the weaver generator to weave practical programs for Capsicum.

The weaver generator solves a version of the policyweaving problem in which an input program, a policy, and the operating system are all modeled as automata. The weaver generator solves such a problem by reducing it to finding a modular winning strategy to a two-player safety game. Intuitively, a two-player safety game is an automaton in which the set of states is partitioned into a set of Attacker states and a set of Defender states. When the game is in an Attacker state, the Attacker can transition the state to any adjacent state, and analogously for the Defender. The goal of the Attacker is to eventually transition the game to an accepting state, and the goal of the Defender is to prevent the Attacker from doing so. A winning Attacker (Defender) strategy is a function that reads the transitions chosen by both the Attacker and Defender and outputs a transition for the Attacker (Defender) such that if the Attacker (Defender) always chooses the transition output by the strategy, then the Attacker (Defender) always wins the game. For a game defined by a pushdown automaton, a modular Attacker (Defender) strategy is a strategy that chooses transitions independently from the transitions chosen before the most recent unmatched push transition. If a game defined by a restricted classes of pushdown automata, called Visibly Pushdown Automata (VPA) [17], has a winning modular Attacker or Defender strategy, then the strategy can be found efficiently [14].

Our policy weaver capweave soundly reduces a policyweaving problem $\operatorname{WeAvE}(P, Q)$ to the problem of finding a winning Defender strategy to a game defined by a VPA. Intuitively, capweave constructs a game in which the choices of an Attacker correspond to instructions that a program can execute, the choices of a Defender correspond to Capsicum primitives that can be invoked, and accepting states are reached when the program violates the policy. A winning Defender strategy for the game thus corresponds to a weaving that ensures that the woven program never violates the policy. The problem of finding a winning Defender strategy is NP-complete in general, but in practice capweave finds a winning strategy to a game efficiently by applying heuristics introduced in previous work ( [15], Sec. 4).

Fig. 7 illustrates how capweave applies the weaver generator. The weaver generator takes as input a program and policy, both represented as VPA's, and an operating

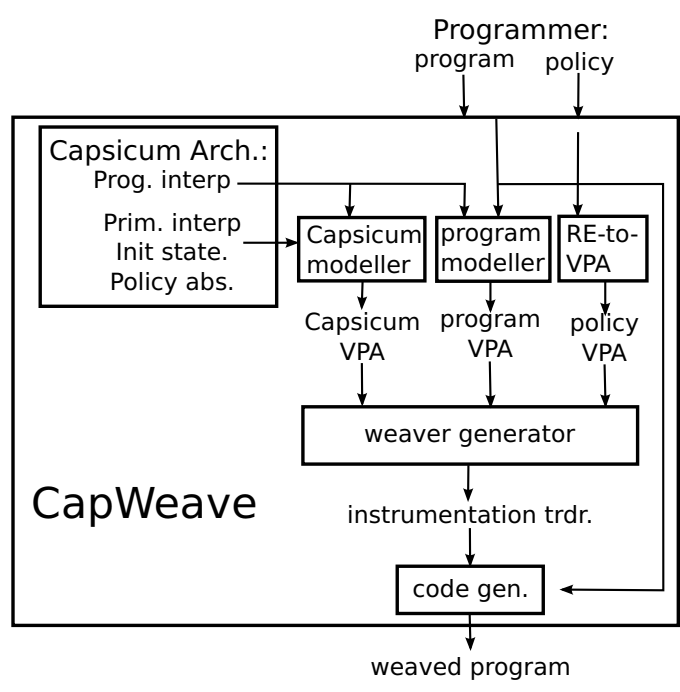

Figure 7. Architecture of capweave. Items in the box labeled "Capsicum Arch." are defined by the Capsicum architect. "Init. state" denotes the initial Capsicum state, "Desc. interp." denotes the interpretation of Capsicum program statements, "Prim. interp" denotes the interpretation of Capsicum primitives, and "Policy abs." denotes the policy abstraction.

system (e.g., Capsicum) modeled as a visibly-pushdown transducer. Thus, to apply the weaver generator, capweave must soundly model its input program, policy, and Capsicum as VPA's. capweave models the program as a VPA constructed directly from the program's interprocedural control-flow graph, which is a standard technique in program analysis [17]. However, in principle, the program can be modeled by any VPA that overapproximates the possible executions of the program (e.g., models constructed via predicate abstraction [18]). Each policy that capweave takes as input is a regular language, so the policy can be represented as a finite-state automaton, and thus as a visibly-pushdown automaton [17]. capweave constructs the transducer model of Capsicum from the space of capability states and Capsicum interpretations (§III-A3). Details of this construction are given in an extended version of this paper [19].

The weaver generator produces an instrumentation strategy represented as a transducer that reads a sequence of program instructions and outputs the next Capsicum primitive that a woven program should execute. capweave compiles such an instrumentation transducer to a woven program by representing the transition function of the instrumentation transducer using a state variable and instructions in the language capinstr (Fig. 4). capweave weaves the input program to consult the state variable to determine which Capsicum primitive to invoke next as the program executes, and then update the state variable. This compilation scheme is described further in the extended version of this paper [19].

If a programmer provides a program $P$ and policy $Q$ for 
which capweave cannot find a solution to $\operatorname{Weave}(P, Q)$, then capweave can, in principle, provide useful diagnostic information to the programmer. There are multiple reasons why capweave may not be able to find a solution to a weaving problem $\operatorname{WeAve}(P, Q):(1) \operatorname{WeAVE}(P, Q)$ may not have a solution. (2) $\operatorname{WeAve}(P, Q)$ may have a solution, but capweave may not find a solution because either (a) when capweave constructs a VPA model of $P$, the resulting model allows $P$ to perform more executions than $P$ can actually perform, or (b) the solution does not correspond to a modular winning Defender strategy [15]. In all cases except (b), the weaver generator produces a winning Attacker strategy that describes the executions that $P$ can perform to violate $Q$, no matter what Capsicum primitives are invoked by any weaving. From such an Attacker strategy and the Capsicum semantics, capweave could construct an unweavable policy $Q^{\prime}$ that is no more restrictive than $Q$ (i.e., $Q^{\prime} \supseteq Q$ ). capweave could then either validate that $Q^{\prime}$ is truly unsatisfiable and provide $Q^{\prime}$ to the user as an explanation of capweave's failure, or use $Q^{\prime}$ to refine its model of the input program. In case (b), capweave could still apply various heuristics to iteratively weaken the input policy, check if the weakened policy has a weaving, and provide this information as diagnostics to the programmer. We have not implemented support for handling failures in capweave, and do not evaluate capweave's usability in the face of such failures. We plan to explore failure diagnostics in future work.

\section{Weaving Practical Programs}

In $\S$ III-A4, we defined the policy-weaving problem for a simple imperative language. However, the weaving problem and our policy weaver can be extended to handle programs written in practical programming languages, such as $\mathrm{C}$, in which programs may have multiple recursive procedures, or manipulate compound datatypes. In particular, capweave weaves programs in the LLVM intermediate language [16].

A key strength of Capsicum is that a program that runs on Capsicum may run code injected by an attacker (e.g., via a stack-smashing attack [20]), and yet can still satisfy a non-trivial security policy. Thus, in practice it is critical that our policy weaver be able to correctly weave programs that can run injected code. We could implement such a weaver by extending the language semantics and policyweaving problem described in $\S \mathrm{III}-\mathrm{A} 2$ and $\S \mathrm{III}-\mathrm{A} 4$ in a straightforward way. The only change we would need to make is that the policy weaver would not be able to choose what Capsicum primitives the woven program will execute after it executes specified program points at which the program might run injected code.

A programmer who uses capweave must understand his program sufficiently well to define a correct policy in terms of program actions paired with capabilities. Furthermore, in practice, the size of a policy may not differ significantly from the size of the code required to instrument the policy. However, the key utility of capweave is that it allows the programmer to reason purely in terms of capabilities that the program must hold over its execution. Because a programmer's ultimate goal is to write a program that holds desired capabilities, this reasoning is strictly easier than determining desired capabilities and then rewriting the program to use the intricate Capsicum primitives to induce the capabilities.

More sophisticated programming tools could further ease the burden of using capweave by, e.g., inferring a likely policy from the callsites of system calls that manipulate descriptors. Such a tool need only determine the capabilities that the program requires as it executes, and discharge to capweave the problem of instrumenting the program to hold the required capabilities. To evaluate fully the utility of capweave and related tools would require a comprehensive programmer study. We leave this as future work.

\section{Practical Experience AND ExPeriments}

We carried out a set of experiments to evaluate the practical utility of capweave. The experiments were designed to answer the following questions:

1) Does capweave allow a programmer to rewrite a program with less effort and with higher assurance than if he manually rewrote the program to invoke Capsicum's primitives?

2) Does capweave rewrite real-world programs to enforce practical policies efficiently?

3) Do programs produced by capweave behave comparably, both in terms of correctness and performance, to programs manually modified by an expert to satisfy the same policy?

To answer these questions, we applied capweave to a set of UNIX utilities-all of which had previously demonstrated security vulnerabilities - so that the instrumented program satisfied a policy that thwarted the vulnerabilities. The programs and policies were derived from either previous work done in developing Capsicum [10], discussions with Capsicum system and application developers on the Capsicum mailing list [13], or collaborative work with security researchers at MIT Lincoln Laboratory (MITLL).

The capweave implementation is $35 \mathrm{k}$ lines of OCaml, and uses the LLVM OCaml API, which corresponds closely to the LLVM API provided for $\mathrm{C}$ and $\mathrm{C}++$. capweave takes as input programs in the LLVM intermediate representation (called bitcode), and outputs instrumented programs as bitcode as well. As a result, it can be inserted into any compiler toolchain that compiles a source program to LLVM bitcode in some intermediate phase.

capweave generates instrumentation code as a multidimensional array that defines what Capsicum primitive should be called as each program point is executed. While the generated code likely cannot be understood easily by a 
programmer, the instrumentation transducer ( $($ III-B) can be recovered from the generated array. The transducer could perhaps be used by a programmer to more easily understand the instrumentation.

The results of our experiments demonstrate that capweave is useful for rewriting programs for Capsicum. In particular:

1) For each of our subject programs, a programmer could apply capweave by annotating their program with only 4-11 lines of code, and writing a policy, expressed purely in terms of Capsicum capabilities, that could be represented with 35-114 lines of our policy language. Thus, capweave can be applied to rewrite programs to satisfy explicit, declarative policies with minimal effort.

2) capweave wove all programs in less than five minutes, except for the PHP CGI interpreter, which took 46 minutes Thus, capweave is efficient enough to be applied to programs in, say, a nightly build system, and in many cases could be integrated into a compiler toolchain used in an edit-compile-run cycle.

3) Programs rewritten by capweave to satisfy a given policy match programs manually written by an expert to satisfy the same policy, and run with overhead within $4 \%$ of unwoven programs on practical workloads. Thus, in practice, capweave produces programs that behave comparably to those written by an expert programmer.

\section{A. Methodology}

To answer the experimental questions presented in $\S \mathrm{IV}$, we applied capweave to weave a set of UNIX utilities as security and performance benchmarks. In this section, we describe each of the benchmarks, and then describe the experiments that we performed on each benchmark.

1) Benchmark Programs and Policies: We now describe each of the benchmark programs and policies used, including its role as a security-critical application, and the source of its policy. While each policy used was inspired by a known vulnerability in the benchmark, each policy restricts the capabilities of large portions of its program's execution. For example, the policies for bzip2 and gzip strongly limit the capabilities of both programs while they execute their compression and decompression functions. Each policy thus potentially mitigates a large class of vulnerabilities that may be unknown when the policy was written. The policy also explicitly describes the limitations of any program rewritten to satisfy the policy, and thus the limits on any attacker who compromises the rewritten program.

bzip2 and gzip: The compression programs bzip2 and gzip can be used by a trusted user to compress data from an untrusted source. On BSD systems, they are often used by root to decompress application packages. The compression and decompression functions of bzip2 and gzip are heavily optimized and quite complex, and have exhibited security vulnerabilities in the past [3], [4]. An attacker who can control the inputs to bzip2 and gzip can craft an input that allows him to execute arbitrary code with the privileges of the user who invoked bzip2 or gzip.

We defined a capweave policy that strictly limits the abilities of an attacker who compromises bzip2 or gzip. The policy restricts bzip2 and gzip to execute with only the capability to read from the source file that holds uncompressed data and write to the file opened to store the compressed output. An attacker who compromises a version of bzip2 or gzip that satisfies such a policy can compromise the integrity of the output files of bzip2 or gzip, but cannot carry out other malicious actions. For instance, the attacker cannot overwrite arbitrary files. Our capweave policies for bzip2 and gzip were inspired by previous work on manually writing programs for Capsicum [10], [21].

php-cgi: Executing programs written in web scripting languages, such as PHP, raises multiple security issues. First, analyzing, monitoring, and restricting the behavior of a program written in a scripting language is inherently difficult. Second, a maliciously-crafted web program can potentially compromise the interpreter that executes it, and then perform any action on its host system that is allowed for the user who launched the interpreter [5].

We defined a capweave policy for the PHP CGI interpreter php-cgi that allows the interpreter to only read from and write to files defined by a small set of simple, easilyaudited checking functions. Our policy strictly limits the file I/O of php-cgi itself, and thus indirectly limits the I/O of any PHP script that the interpreter executes. We defined the policy by collaborating with a group of researchers from MITLL.

tar: The tar archiving utility archives sets of files into a single file. Unfortunately, past versions of tar have contained vulnerabilities that allow an attacker who controls the inputs to tar to run injected code with the privileges of the user who invoked tar [6], [7].

We defined a capweave policy that strictly limits the abilities of an attacker who compromises tar. The policy restricts tar to execute vulnerable functions without ambient authority. An attacker who compromises a version of tar that satisfies such a policy can compromise the integrity of output files opened by tar, but cannot carry out other malicious actions.

tcpoump: tcpoump is a widely-used network-facing application that historically has been the target of many exploits. tcpdump takes as input a Berkeley Packet Filter (BPF), and a device from which to read packets. In a correct execution, it reads packets from the device, matches them against the input BPF, and if the packet matches, prints the packet to standard output. Unfortunately, the packetmatching code in tcpdump is complex; in previous versions of tcpdump, an attacker who controls the network input to 
tcpdump can craft a packet that allows him to take control of the process executing tcpdump [1].

We defined a capweave policy for tcpdump that strictly limits the power of an attacker who is able to compromise tcpdump. In previous work on Capsicum [10], the Capsicum developers instrumented tcpdump so that it could only read from its input network device and write to standard output. The Capsicum developers later found through testing that this instrumentation did not allow tcpdump to resolve network addresses in a packet, and the developers revised the instrumentation of tcpdump so that only a small, trusted DNS resolver could open files. The capweave policy for tcpdump describes the policy that the revised instrumentation satisfies.

wget: The wget downloader, its vulnerabilities, and its capweave policy were discussed in §II. Our policy for wget was inspired by discussion on the Capsicum-developer mailing list and known vulnerabilities of wget [2], [13].

\section{B. Experimental Procedure}

For each of the benchmark programs and policies described in $\S \mathrm{IV}$-A1, we defined the benchmark's policy in the capweave policy language, and applied capweave to the program and policy. We also obtained a version of each program that was manually modified to satisfy the policy. In previous work, the Capsicum developers manually rewrote bzip2, gzip, and tcpdump to satisfy informal versions of the policies described in $§ \mathrm{IV}-\mathrm{A} 1$. We recompiled these versions with the LLVM compiler so that we could compare their runtime overhead with the runtime overhead of the programs woven by capweave. We manually instrumented the other three benchmarks to satisfy each of their policies. The woven and manually instrumented programs were compiled with the default optimization of each benchmark ("-O2" for each program). capweave was applied to optimized LLVM bitcode.

We ran bzip2, gzip, php-cgi, tcpdump, and wget on the test workloads included in the source distribution of each program. We ran tar to archive its own source directory. We ran each original benchmark, woven benchmark, and manually rewritten benchmark on the benchmark's test suite, and compared the executions. In particular, we counted the number of tests that each of the benchmark programs passed, and measured the runtime performance overhead of the rewritten programs compared to the original benchmark. Because the total time taken by each benchmark on the test workloads supplied with the source was often less than a second, we also measured performance on larger, more realistic workloads.

To validate that the woven programs mitigated attacks according to their policy, we introduced into each program a "backdoor" that attempted to carry out an attack disallowed by the program's policy. We then ran the woven program on an input that triggered the backdoor, and observed that the goal of the attack was not achieved.

\section{Analysis of Results}

The results of our experiments are given in Tab. I. (The performance numbers reported in Tab. I are from the test workloads included in the source distribution of each program. Performance on larger, more realistic workloads is discussed below.) For each benchmark, Tab. I contains three groups of measurements of our experience weaving the benchmark. The first group (cols. 3-5) measures the complexity of the policy for which the benchmark was woven, and contains the number of lines of code in which each policy is represented in our policy language, as well as the size of the policy DFA constructed by capweave. The number of lines of policy-language code ("Lines") indicates that the policy language supported by capweave can express practical policies concisely.

The second group of measurements in Tab. I (cols. 6-7) measures the performance of capweave, and contains the time and peak memory used by capweave. Each benchmark was woven on a server that has sixteen $2.4 \mathrm{GHz}$ cores and 32 GB of memory, although the capweave implementation executes sequentially. The running time (cf. "Weaving Time") and peak memory (cf. "Weaving Mem.") indicate that capweave could be included in the edit-compile-run toolchain of many programs of small-to-medium size, and could be included in the nightly build system of a program of large size. The running time and peak memory also indicate that the performance of capweave is strongly determined by the size of the policy, in particular the size of the policyautomaton alphabet, more than the size of the input program (cf. "States", "Alpha. Size", "Weaving Time", and "Weaving Mem").

The third group of measurements in Tab. I (cols. 815) measures the performance of programs rewritten by capweave, and contains the number of reference tests that the unwoven (i.e., baseline) program passed ("Tests"), the number of tests passed by the woven version of each benchmark ("OK"), the number of program points at which capweave added instrumentation ("Woven Points"), the number of functions that the benchmark executes in a synchronous fork ("Interproc. Funcs."), the runtime of the baseline program ("Base"), the runtime of the woven program expressed as a multiple of the runtime of the baseline ("Woven Overhead"), the runtime of the handwoven program as a multiple of the runtime of the baseline ("Hand Overhead"), and the percentage overhead of the runtime of the woven program over the runtime of the handwoven program ("capweave/ Hand"). The geometric mean of all "capweave/ Hand" values is 1.298 (i.e., capweave overhead is $29.8 \%$ ). Each benchmark was run on a host machine with eight $2.2 \mathrm{GHz}$ processors and $6 \mathrm{~GB}$ of 


\begin{tabular}{|c|c|c|c|c|c|c|c|c|c|c|c|c|c|c|}
\hline \multicolumn{2}{|c|}{ Program Features } & \multicolumn{3}{|c|}{ Policy Size } & \multicolumn{2}{|c|}{ Weaver } & \multicolumn{8}{|c|}{ Woven-Program Performance } \\
\hline Prog. Name & $\mid \begin{array}{c}\text { Size } \\
(\mathrm{KLOC})\end{array}$ & Lines & States & $\begin{array}{l}\text { Alpha. } \\
\text { Size }\end{array}$ & $\begin{array}{c}\text { Weaving } \\
\text { Time }\end{array}$ & $\begin{array}{l}\text { Weaving } \\
\text { Memory }\end{array}$ & Tests & $\mathrm{OK}$ & $\begin{array}{l}\text { Woven } \\
\text { Points }\end{array}$ & $\begin{array}{c}\text { Interproc. } \\
\text { Funcs. }\end{array}$ & Base (s) & $\begin{array}{l}\text { Woven } \\
\text { Overhd. }\end{array}$ & $\begin{array}{c}\text { Hand } \\
\text { Overhd. }\end{array}$ & \begin{tabular}{|c|} 
capweaved \\
Hand $(\%)$
\end{tabular} \\
\hline bzip2-1.0.6 & 8 & 70 & 5 & 5,156 & $4 \mathrm{~m} 57 \mathrm{~s}$ & $0.3 \mathrm{~GB}$ & 6 & 6 & 66 & 1 & 0.593 & 1.099 & 0.909 & 20.90 \\
\hline gzip-1.2.4 & 9 & 68 & 5 & 1,787 & $3 \mathrm{~m} 26 \mathrm{~s}$ & $0.2 \mathrm{~GB}$ & 2 & 2 & 55 & 1 & 0.036 & 1.278 & 1.111 & 15.03 \\
\hline php-cgi-5.3.2 & 852 & 114 & 11 & 15,777 & $46 \mathrm{~m} 36 \mathrm{~s}$ & $25.3 \mathrm{~GB}$ & 11 & 2 & 213 & 2 & 0.289 & 1.938 & 1.170 & 65.64 \\
\hline $\operatorname{tar}-1.25$ & 108 & 49 & 8 & 143 & $0 \mathrm{~m} 08 \mathrm{~s}$ & $0.2 \mathrm{~GB}$ & 1 & 1 & 62 & 2 & 0.156 & 21.917 & 13.301 & 64.78 \\
\hline t cpdump-4.1.1 & 87 & 52 & 6 & 223 & $0 \mathrm{m09s}$ & $0.3 \mathrm{~GB}$ & 29 & 27 & 88 & 1 & 1.328 & 1.224 & 0.981 & 24.77 \\
\hline wget-1.12 & 64 & 35 & 3 & 549 & $0 \mathrm{~m} 10 \mathrm{~s}$ & $0.2 \mathrm{~GB}$ & 4 & 4 & 246 & 1 & 4.539 & 1.106 & 1.096 & 0.91 \\
\hline
\end{tabular}

Table I

EXPERIMENTAL DATA FOR A SET OF BENCHMARKS PROGRAMS AND POLICIES. THE FIELDS OF THE TABLE ARE DISCUSSED IN $\S$ IV-C. IN THIS TABLE, THE PERFORMANCE NUMBERS REPORTED ARE THOSE FROM THE TEST WORKLOADS INCLUDED IN THE SOURCE DISTRIBUTION OF EACH PROGRAM. PERFORMANCE ON A LARGER, MORE REALISTIC WORKLOAD IS DISCUSSED IN $\S$ IV-C.

memory, in a Capsicum virtual machine with one processor and 2 GB of memory.

Each woven program behaved identically to its corresponding hand-woven program on each test, and behaved identically to its corresponding unwoven program on each test, except for some tests included with tcpdump and php-cgi. The woven tcpdump failed tests included with tcpdump that gave a filepath to a file containing a secret key for decrypting IPsec ESP packets, instead of giving the secret key directly on the command line. The tcpdump policy specified that the woven tcpdump should not be able to open any file except for the input network device, and hence could not open the file containing the secret key. The woven php-cgi failed tests included with php-cgi because no weaving of php-cgi can simultaneously satisfy the policy specified by the MITLL group and pass all of the tests. For example, the MITLL policy does not allow a PHP program to create a new file in its current directory. The woven php-cgi thus failed any test in which a program tried to create a file for output in its current directory. tcpdump and php-cgi thus illustrate one key aspect of capweave: capweave allows a programmer to specify the capabilities that a program should hold throughout an execution, and automatically obtain a program that holds the specified capabilities. However, a programmer still must determine manually whether the capabilities specified by a particular policy strike an acceptable balance between the security requirements of the program and its original functionality.

The number of program points at which capweave introduced instrumentation (i.e., "Woven Points") was small relative to the size of each benchmark. Furthermore, the number of functions that each woven benchmark executed between a synchronous fork and join (i.e., "Interproc. Funcs") was small, and matched the number of functions that each hand-woven benchmark executed between a synchronous fork and join (and thus likely was minimal). However, woven versions of bzip2, gzip, php-cgi, and wget incurred noticeable overhead. We suspected that the woven versions of these programs would introduce the most overhead on small workloads, because on such workloads, the fixed overhead of executing a synchronous fork and join dominates the overall runtime of the program.

To measure the performance of the woven programs on larger workloads, we generated a $1 \mathrm{~GB}$ file of source code from the Capsicum kernel source tree, and used it as a workload for bzip2, gzip, and wget. The unwoven bzip2 compressed the large file in $25 \mathrm{~m} 31 \mathrm{~s}$, and the woven bzip2 compressed the large data with $4 \%$ overhead over the baseline time. The unwoven $g z$ ip compressed the large file in $5 \mathrm{~m} 27 \mathrm{~s}$, and the woven gzip compressed the large data with $3 \%$ overhead over the baseline time. The unwoven wget downloaded the large file from a server on the same local network in $1 \mathrm{~m} 06 \mathrm{~s}$, and the woven wget downloaded the large data with $-4 \%$ overhead over the baseline time, indicating that the overhead of the weaving is obscured by noise introduced by network traffic. Thus, the maximum overhead of the woven programs over unwoven programs is $4 \%$, and geometric mean of all the overheads is $1 \%$. The overhead for php-cgi depends entirely on how frequently an input PHP script opens files over the course of its execution.

The woven versions of tar and tcpdump introduced noticeable overhead on operations that execute frequently on all workloads, such as a procedure in tar that reads data into a buffer, or a procedure in tcpdump that resolves network addresses to names. The per-operation overhead induces an enormous overhead in tar in particular, and illustrates another limitation of capweave: some policies induce capweave to introduce costly primitives, such as fork, at program points that induce considerable overhead, whereas capweave might be able to instrument other program points that induce much less overhead. The overhead of the hand-woven program, while less than the overhead of the capweave-woven program, is still considerable: in our experience, weaving tar efficiently is a difficult problem, and one that could benefit significantly from further automatic-tool support. In future work, we hope to address this limitation by extending capweave to use a cost metric, and generalizing the game solver to find optimal strategies 
for quantitative games [22].

\section{RELATED WORK}

Capability systems: Karger [23] introduced a capability system that mitigates the effects of an attack by a malicious program run on the system. The Capsicum operating system [10] provides security primitives to support isolating components of a program in sandboxes that run with different capabilities based on UNIX file descriptors. This paper describes the capweave tool, which greatly eases the burden of using Capsicum by allowing policies to be stated as a separate specification that capweave weaves into the program automatically.

Security monitors: Operating systems that provide security system calls, such as Capsicum, HiStar [11], Wedge [24] etc., allow an application developer to define program-specific policies (where the nature of the policy depends on the security primitives offered by the operating system). In contrast, Mandatory Access Control (MAC) operating systems, such as [25]-[27] only support system-wide policies described in terms of standard system events. Such policies cannot refer to important events in the execution of a particular program, but many practical policies can only be defined in terms of such events [28]. UNIX can monitor programs to ensure that they satisfy policies if the program correctly uses the setuid system call, but in general this approach suffers the same shortcomings as MAC systems. In comparison, systems with security primitives allow an application to signal key events in its execution to the operating system. Watson has described the challenges of developing an access-control system, and has surveyed recent implementations of such systems [29].

An Inline Reference Monitor (IRM) rewriter takes a policy expressed as an automaton and instruments a target program with an IRM, which executes in the same memory space as the program, and halts the program if it attempts to perform some sequence of actions that would violate the policy [28], [30]. Edit automata [31] generalize IRMs by also supressing or adding security-sensitive events to ensure that the program satisfies a policy. Because an IRM (or edit automaton) executes in the same memory space as the program that it monitors, it can enforce policies defined over arbitrary events in the execution of the program. However, for the same reason, an IRM can only monitor the execution of managed code. In comparison, systems with security primitives can safely and efficiently monitor programs composed largely of unmanaged code [10], [11].

Writing programs for security monitors: Prior work on programming aids for systems with security primitives automatically verifies that a program instrumented to use the Flume OS [9] primitives enforces a high-level policy [32], automatically instruments programs to use the primitives of the HiStar OS to satisfy a policy [33], and automatically instruments programs [32] to use the primitives of the Flume
OS [9]. However, the languages of policies used in the approaches presented in [33], [34] are not temporal, and cannot clearly be applied to other systems with security primitives. The weaving algorithm presented in this paper applies a known automata-theoretic weaving algorithm [15], and can, in principle, be applied in multiple settings. The main contribution of this paper is to describe how the automata-theoretic algorithm can be applied as an engine to rewrite programs for a practical capability system.

In the privsep project [35], OpenSSH was rewritten manually to execute using a trusted, privileged parent process and an unprivileged child process. A programmer can use the Privman [36] library to manually compartmentalize a UNIX daemon into high and low-privilege processes. Previous work [37], [38] automatically partitions programs so that high and low-confidentiality data are processed by separate processes, or on separate hosts. The SOAPP project [39] proposes a semi-automatic technique in which a programmer annotates a program with a hypothetical sandbox, and a program analysis validates that the sandbox does not introduce unexpected program behavior. The SOAPP approach is similar in spirit to [32], which uses model checking to verify that a programmer-proposed partitioning and set of calls to security primitives satisfies a given policy. In contrast, capweave automatically infers where to invoke library functions that cause the program to execute in different processes (if necessary), and rewrites the program accordingly.

Skalka and Smith [40] present an algorithm that takes a Java program instrumented with capability-based security checks, and attempts to show statically that some checks are always satisfied. Hamlen et al. [41] verify that programs rewritten by an IRM rewriter are correct. Thus, the work in both of those papers concerns identifying superfluous capability checks in managed programs, whereas our work concerns how to infer the correct placement of primitives to restrict the capabilities of unmanaged programs.

Safety games: Safety games have been studied as a framework for synthesizing reactive programs and control mechanisms [14], [42]. Previous work describes algorithms that take a safety game, determine which player can always win the game, and synthesize a winning strategy for the winning player [14]. The key contribution of our work is to demonstrate that such game-theoretic problems can be applied in practice to rewrite programs to enforce a security policy.

\section{CONCLUSION}

New operating systems, such as the Capsicum capability system, define powerful system-level primitives for secure programming, but such primitives are non-trivial to use. This paper presents a policy-weaver for Capsicum, capweave, that takes from a programmer an uninstrumented program and a high-level policy that describes correct behavior of the 
program. capweave automatically infers where to invoke security primitives and rewrites the program accordingly. In practice, capweave produces programs that match the behavior and performance of programs manually modified by an expert. capweave is designed so that a Capsicum Architect can easily add, remove, or update new programming libraries as they continue to be developed.

Acknowledgments: We gratefully acknowledge the work of the Capsicum development team, in particular Pawel Dawidek, Khilan Gudka, and Ben Laurie, in developing Capsicum and manually instrumenting programs for Capsicum. We thank Michael Zhivich and Jeffrey Seibert at MITLL for developing the capweave policy for PHP. We thank our shepherd, Niels Provos.

Supported, in part, by DARPA and AFRL under contracts FA8650-10-C-7088 and FA8750-10-C-0237. The views, opinions, and/or findings contained herein are those of the authors and should not be interpreted as representing the official views or policies, either expressed or implied, of the Defense Advanced Research Projects Agency or the Department of Defense.

\section{REFERENCES}

[1] “CVE-2007-3798," http://cve.mitre.org/cgi-bin/cvename.cgi? name $=$ CVE-2007-3798, July 2007.

[2] “CVE-2004-1488," http://cve.mitre.org/cgi-bin/cvename.cgi? name $=$ CAN-2004-1488, Feb 2005.

[3] “CVE-2010-0405," http://cve.mitre.org/cgi-bin/cvename.cgi? name $=$ CVE-2010-0405, April 2010.

[4] "Vulnerability note VU\#381508," http://www.kb.cert.org/ vuls/id/381508, July 2011.

[5] "Vulnerability note VU\#520827," http://www.kb.cert.org/ vuls/id/520827, May 2012.

[6] "CVE-2007-4476," http://cve.mitre.org/cgi-bin/cvename.cgi? name $=$ CVE-2007-4476, Aug 2007.

[7] "GNU Tar and GNU Cpio rmt_read_() function buffer overflow," http://xforce.iss.net/xforce/xfdb/56803, Mar 2010.

[8] P. Efstathopoulos, M. Krohn, S. VanDeBogart, C. Frey, D. Ziegler, E. Kohler, D. Mazières, F. Kaashoek, and R. Morris, "Labels and event processes in the Asbestos operating system," in SOSP, 2005.

[9] M. Krohn, A. Yip, M. Brodsky, N. Cliffer, M. F. Kaashoek, E. Kohler, and R. Morris, "Information flow control for standard OS abstractions," in SOSP, 2007.

[10] R. N. M. Watson, J. Anderson, B. Laurie, and K. Kennaway, "Capsicum: Practical capabilities for UNIX," in USENIX Security, 2010.

[11] N. Zeldovich, S. Boyd-Wickizer, E. Kohler, and D. Mazières, "Making information flow explicit in HiStar," in OSDI, 2006.

[12] "FreeBSD 9.0-RELEASE announcement," http: //www.freebsd.org/releases/9.0R/announce.html, Jan. 2012.

[13] "cl-capsicum-discuss - Capsicum project discussion list," https://lists.cam.ac.uk/mailman/listinfo/cl-capsicum-discuss, 2012.

[14] R. Alur, S. L. Torre, and P. Madhusudan, "Modular strategies for recursive game graphs," in TACAS, 2003.

[15] W. R. Harris, S. Jha, and T. W. Reps, "Secure programming via visibly pushdown safety games," in $C A V, 2012$.

[16] C. Lattner, http://llvm.org/, Nov. 2011.
[17] R. Alur and P. Madhusudan, "Visibly pushdown languages," in STOC, 2004.

[18] S. Graf and H. Saïdi, "Construction of abstract state graphs with PVS," in $C A V, 1997$.

[19] W. R. Harris, S. Jha, T. Reps, J. Anderson, and R. N. M. Watson, "Declarative, temporal, and practical programming with capabilities," http://minds.wisconsin.edu/handle/1793/64927, University of Wisconsin-Madison, Tech. Rep. TR1785, 2013.

[20] A. One, "Smashing the stack for fun and profit," Phrack Magazine, vol. 49, no. 14, 1998.

[21] "Using Capsicum for sandboxing," http://www.links.org/?p= 1242, April 2012.

[22] A. Ehrenfeucht and J. Mycielski, "Positional strategies for mean payoff games," International Journal of Game Theory, vol. 8, no. 2, 1979.

[23] P. A. Karger, "Limiting the damage potential of discretionary trojan horses," in IEEE $S \& P, 1987$.

[24] A. Bittau, P. Marchenko, M. Handley, and B. Karp, "Wedge: Splitting applications into reduced-privilege compartments," in NSDI, 2008

[25] P. Loscocco and S. Smalley, "Integrating flexible support for security policies into the Linux operating system," in USENIX Annual Technical Conference, 2001.

[26] O. S. Saydjari, "Lock : An historical perspective," in ACSAC, 2002.

[27] C. Wright, C. Cowan, J. Morris, and S. S. G. Kroah-Hartman, "Linux security modules: General security support for the Linux kernel," in Found. of Intrusion Tolerant Systems, 2003.

[28] U. Erlingsson and F. B. Schneider, "IRM enforcement of Java stack inspection," in IEEE $S \& P, 2000$.

[29] R. N. M. Watson, "A decade of OS access-control extensibility," Commun. ACM, vol. 56, no. 2, Feb. 2013.

[30] M. Abadi, M. Budiu, Ú. Erlingsson, and J. Ligatti, "Controlflow integrity," in CCS, 2005.

[31] J. Ligatti, L. Bauer, and D. Walker, "Edit automata: Enforcement mechanisms for run-time security policies," Int. J. Inf. Sec., vol. 4, no. 1-2, 2005.

[32] W. R. Harris, N. A. Kidd, S. Chaki, S. Jha, and T. Reps, "Verifying information flow control over unbounded processes," in $F M, 2009$.

[33] P. Efstathopoulos and E. Kohler, "Manageable fine-grained information flow," in EuroSys, 2008.

[34] W. R. Harris, S. Jha, and T. Reps, "DIFC programs by automatic instrumentation," in CCS, 2010.

[35] N. Provos, "Privilege separated OpenSSH," http://www.citi. umich.edu/u/provos/ssh/privsep.html, Aug 2003.

[36] D. Kilpatrick, "Privman: A library for partitioning applications," in USENIX Annual Technical Conference, 2003.

[37] D. Brumley and D. X. Song, "Privtrans: Automatically partitioning programs for privilege separation," in USENIX Security Symposium, 2004.

[38] S. Chong, J. Liu, A. C. Myers, X. Qi, K. Vikram, L. Zheng, and $\mathrm{X}$. Zheng, "Secure web application via automatic partitioning," in SOSP, 2007.

[39] K. Gudka, R. N. M. Watson, S. Hand, B. Laurie, and A. Madhavapeddy, "Exploring compartmentalization hypothesis with SOAPP," in AHANS 2012, 2012.

[40] C. Skalka and S. F. Smith, "Static enforcement of security with types," in ICFP, 2000.

[41] K. W. Hamlen, G. Morrisett, and F. B. Schneider, "Certified in-lined reference monitoring on .NET," in PLAS, 2006.

[42] R. Alur, T. A. Henzinger, and O. Kupferman, "Alternatingtime temporal logic," in FOCS, 1997. 